\title{
Comparaison de deux modèles d'enseignement de la chimie en $5^{\text {ème }}$
}

\section{Erick Cazalet et L.Y. Chen}

\section{(2) OpenEdition \\ 12 Journals}

Édition électronique

URL : http://journals.openedition.org/trema/2336

DOI : $10.4000 /$ trema.2336

ISSN : 2107-0997

\section{Éditeur}

Faculté d'Éducation de l'université de Montpellier

\section{Édition imprimée}

Date de publication : 1 mai 1993

Pagination : 69-76

ISSN : 1167-315X

\section{Référence électronique}

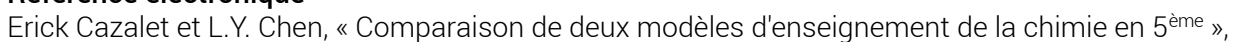
Tréma [En ligne], 3-4 | 1993, mis en ligne le 01 mai 1993, consulté le 01 mai 2019. URL : http:// journals.openedition.org/trema/2336 ; DOI : 10.4000/trema.2336

Ce document a été généré automatiquement le 1 mai 2019.

Trema 


\title{
Comparaison de deux modèles d'enseignement de la chimie en 5 ème
}

\author{
Erick Cazalet et L.Y. Chen
}

1 Un enseignement peut se définir comme un mode d'intervention qui tente de se régler sur le fonctionnement intellectuel de l'élève afin de mieux réussir le dépassement des difficultés et la modification des conceptions.

2 Le savoir enseigné, différent du savoir savant, doit tenir compte du niveau de développement cognitif des élèves. Il nécessite une transposition des formulations conceptuelles en fonction de ce développement.

3 L'élève, avant qu'on lui enseigne, dispose de structures intellectuelles, de connaissances préexistantes relativement résistantes aux apprentissages. Cette structure d'accueil, résultant des expériences de la vie quotidienne et des coordinations des actions de l'enfant lui-même, a souvent peu de rapport avec la structure des disciplines scientifiques. Pour comprendre l'efficacité d'un enseignement, il nous semble nécessaire d'explorer et de connaître ces connaissances préexistantes telles qu'elles sont et non telles qu'on prétend les édifier a priori.

4 Dans cette communication, nous relaterons une expérience d'enseignement concrète menée en classe de cinquième au collège de Sommières (Gard). Cette communication fait suite à une recherche de Psychologie Cognitive.

\section{Les modèles d'enseignement}

5 Le schéma suivant (figure 1) est proposé par Martinand (1985) pour étudier le concept d'élément chimique en classe de 5ème. 
Figure 1. : Schéma d'étude du concept d'élément.

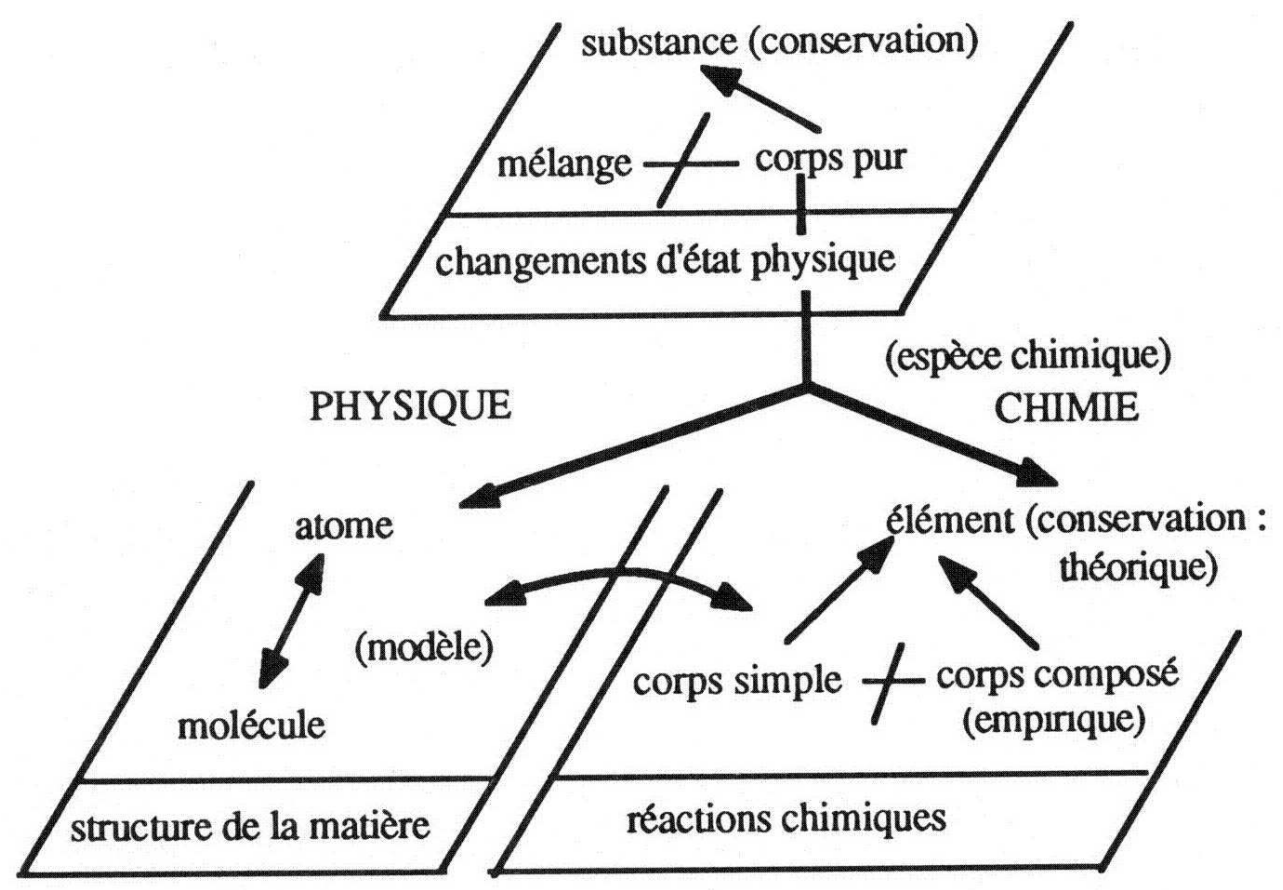

6 L'interprétation que nous faisons de ce schéma a conduit à élaborer deux modèles et deux contenus d'enseignement préalables à l'étude du cycle des réactions chimiques du soufre : un enseignement sur une base expérimentale désigné par $5 \mathrm{E}$, un enseignement sur une base théorique (le modèle particulaire) désigné par 5P. Ces deux enseignements précèdent un enseignement commun, indifférencié qui s'appuie sur une série d'expériences: celles du "cycle des réactions chimiques du soufre", prévu par les programmes et instructions officielles. Cela concerne une première approche de la permanence des éléments chimiques, une première approche de la conservation des atomes au cours d'une réaction chimique. Nous avons cherché à mieux comprendre les effets respectifs des deux enseignements préalables (5E et $5 \mathrm{P}$ ), non seulement sur les apprentissages liés aux enseignements communs mais aussi sur des représentations plus générales.

\section{L'enseignement sur la base expérimentale $(5 \mathrm{E})$}

7 Aux classes expérimentales notées $5 \mathrm{E}$, l'enseignement commence par l'étude de trois réactions chimiques en référence aux contenus officiels des programmes:

1. « combustion du fer dans l'oxygène. »

2. « conséquence sur la constitution de l'air par action de la limaille de fer à froid, la rouille. »

3. "réaction entre le fer et l'acide chlorhydrique " (test à l'eau de javel pour retrouver l'élément fer.).

8 Suivent les quatre expériences «sur les réactions chimiques du soufre (comme pour l'enseignement de 5P). Résumons cela par :

1. $\mathrm{Fe}+\mathrm{S} \rightarrow \mathrm{FeS}$

2. $\mathrm{FeS}+2 \mathrm{HCl} \rightarrow \mathrm{FeCl} 2+\mathrm{H} 2 \mathrm{~S}$;

3. $\mathrm{H} 2 \mathrm{~S}+1 / 2 \mathrm{O} 2 \rightarrow \mathrm{H} 2 \mathrm{O}+\mathrm{S}$; 
4. Test à l'eau de javel sur la solution de FeCl2.

9 Ces quatre expériences sont conduites avec la même méthode expérimentale que celle du modèle 5P.

10 L'enseignement selon la base particulaire (5P)

11 Dans un premier temps, toujours relativement aux programmes et instructions de la classe de 5ème, l'enseignement porte pour les 5P d'abord sur trois chapitres.

1. «Discontinuité de la matière; le modèle particulaire. »

2. «Atomes, molécules : existence, dimension, symbole, formule.»

3. «Modèle particulaire du corps, simple ou composé, et du mélange dans le cas de corps à l'état gazeux. »

12 Cet enseignement est fait d'informations données par le maître, de travaux sur documents (livre de 5ème), de discussions. Les élèves suivent alors les quatre expériences sur les réactions chimiques du soufre (comme pour l'autre enseignement $5 \mathrm{E}$ ).

1. $\mathrm{Fe}+\mathrm{S} \rightarrow \mathrm{FeS}$;

2. $\mathrm{FeS}+2 \mathrm{HCl} \rightarrow \mathrm{FeCl} 2+\mathrm{H} 2 \mathrm{~S}$;

3. $\mathrm{H} 2 \mathrm{~S}+1 / 2 \mathrm{O} 2->\mathrm{H} 2 \mathrm{O}+\mathrm{S}$;

4. Test à l'eau de javel sur la solution de $\mathrm{FeCl} 2$.

\section{Remarque}

Compte tenu des conditions matérielles et de sécurité - nous travaillons avec des classes de 25 à 28 élèves - c'est le maître qui a procédé aux expériences devant les élèves. Les discussions, commentaires et compte rendu sur le cahier sont menés parallèlement avec la classe entière. Cela confère à notre étude une valeur de sondage seulement.

\section{Déroulement de l'enseignement et de son évaluation}

14 Premier temps: tous les sujets passent un prétest. Il s'agit de permettre aux élèves d'exprimer leurs représentations naïves, leurs modèles à propos de divers phénomènes à partir des questions posées.

15 Deuxième temps : les élèves suivent un enseignement préparatoire, contenus, démarches et activités différentes selon $5 \mathrm{E}$ ou $5 \mathrm{P}$. Les apprentissages ne seront pas évalués ici. Ce sont leurs effets sur le troisième temps que nous analyserons.

16 Troisième temps: c'est le moment de l'étude du cycle des réactions chimiques. Les expériences sont montrées puis résumées avec des schémas. Au fur et à mesure de la présentation des schémas, des questionnaires à choix multiples sont proposés (QCM 1 , QCM 2 et QCM 3).

17 Quatrième temps : c'est le temps du postest. Tous les élèves subissent une nouvelle fois le questionnaire initial afin de cerner les modifications dans les représentations.

\section{Population}

18 Le tableau I indique la distribution de notre population. Ces élèves sont issus de classes différentes. 
Tableau I - Distribution de la population.

\section{5ème \\ Expérimental}

Garçons

Filles

Total
7

8

15 5ème

Particule

7

8

15

Trente élèves de 5 ème choisis aléatoirement dans 4 classes de 25 à 28 élèves. L'âge moyen est 12,9 ans. Ces 30 élèves sont répartis en deux grands groupes de 15 . Avec un groupe l'enseignement aura une base seulement expérimentale (5E). Avec l'autre groupe l'enseignement prendra appui sur le modèle particulaire (5P).

\section{Résultats}

\section{Résultats du bilan d'apprentissage}

Les tableaux qui suivent, résument les résultats significatifs de ce bilan.

- QCM 1.Q2 : Mettons de la poudre de soufre et de la poudre de fer ensemble. On obtient un mélange ou une liaison chimique? La bonne réponse est ?mélange". Résultat: (cf tableau II)

Les élèves de $5 \mathrm{E}$ tendent à penser à la réaction chimique à la place du mélange. Les élèves de $5 \mathrm{P}$ tendent à indifférencier la réaction chimique et le mélange.

Tableau II-QCM 1.

$\begin{array}{ccccc}\text { Mélange } & \begin{array}{c}\text { Réaction } \\ \text { chimique }\end{array} & \begin{array}{c}\text { Indiffé- } \\ \text { renciation }\end{array} & \begin{array}{c}\text { Autres } \\ \text { réponses }\end{array} \\ \text { 5èmeE } & 10 & 4 & 0 & 1 \\ \text { 5èmeP } & 9 & 0 & 4 & 2\end{array}$

$$
(\chi 2=9,05 ; \text { seuil significatif }=0,02)
$$

Le tableau II nous indique que $5 \mathrm{E}$ et $5 \mathrm{P}$ se différencient par les mauvaises réponses.

24 III). Les élèves de la classe 5P ayant répondu «le soufre » (la bonne réponse) sont plus nombreux que ceux de la classe $5 \mathrm{E}$. 


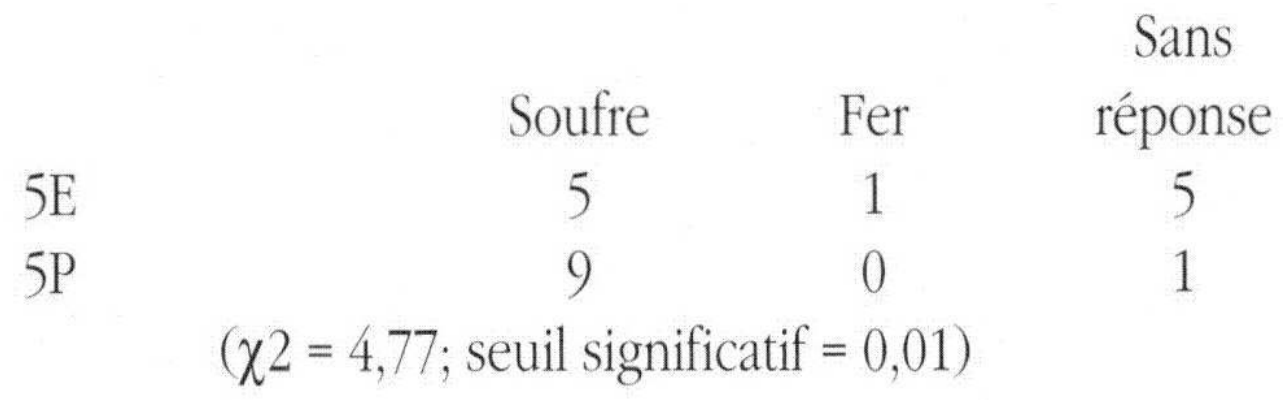

- QCM3.Q14 : Le soufre était là seulement au début et disparaît ensuite? Résultat : (cf tableau IV). Les élèves de la classe 5P ayant répondu ?non" (la bonne réponse) sont plus nombreux que ceux de la classe $5 \mathrm{E}$.

Tableau IV. QCM 3
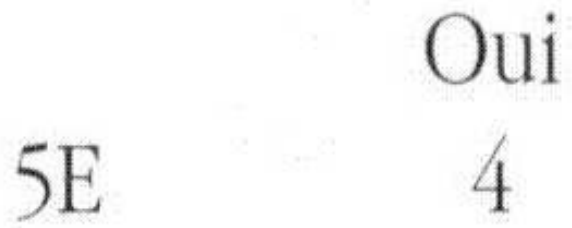

$5 \mathrm{P}$

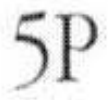

$(\chi 2$

$=2,6$; seuil significatif $=0,10$ )
A propos de ce bilan d'apprentissage, QCM2 et QCM3 sont les mêmes questions posées deux fois. La première fois pour l'élément fer, la deuxième fois pour l'élément soufre. Si on examine la cohérence des réponses dans ces questions, on pourrait confirmer ou infirmer la stabilité des acquisitions dans les deux groupes d'élèves. Les résultats montrent que des réponses des élèves de $5 \mathrm{P}$ sont plus cohérentes que celles de $5 \mathrm{E}$ dans le QCM 2. (cf tableau V).

Voici la question de Q2 (et de Q7) : «le soufre (le fer) en poudre a beaucoup changé au cours des expériences? Est-il devenu autre chose que du soufre (fer) qui contient le soufre (fer) ? oui/non? » (cf. tableau VI) 


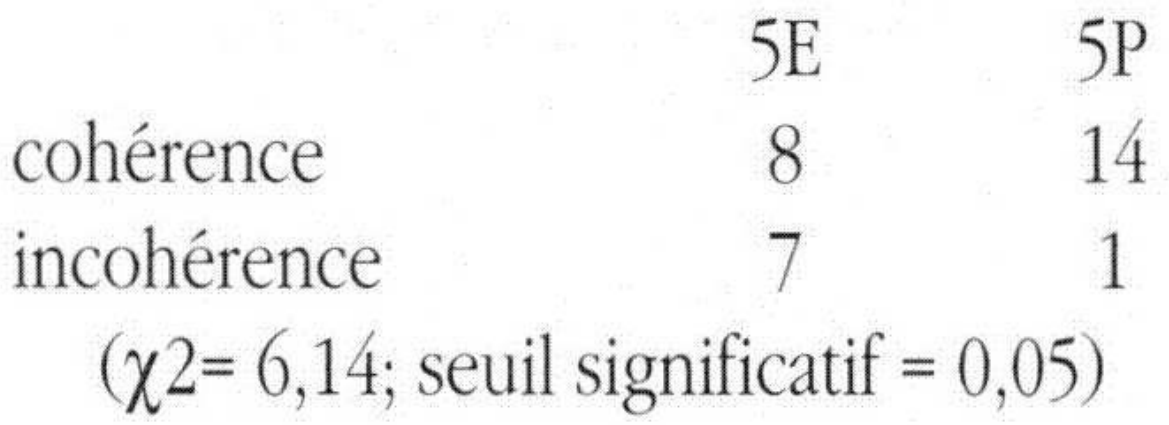

Tableau VI - Cohérence des réponses en fonctions des erreurs et des réussites.

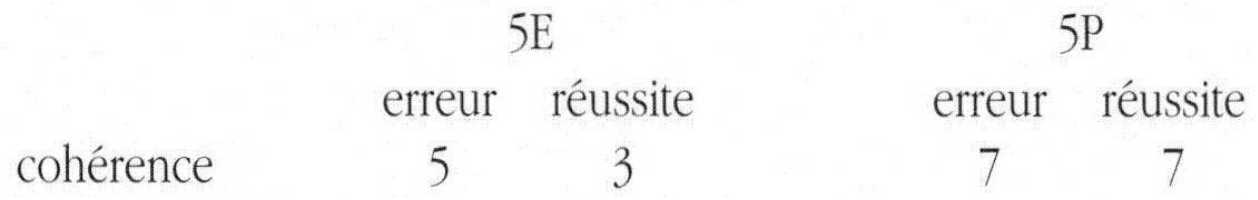

(réponses cohérentes: celles qui sont «oui » ou «non» pour ces deux questions; réponses incohérentes: celles qui sont « oui » dans une question et «non» dans l'autre.)

\section{Résultats au questionnaire général (prétest-postest)}

Les différences et les évolutions qui portent sur le dessin d'air et la définition de molécule. 
Tableau VII - Résultats comparés prétest/postest : dessin d'air.

\begin{tabular}{|c|c|c|c|c|c|c|c|c|c|}
\hline \multicolumn{5}{|c|}{ Prétest } & \multicolumn{5}{|c|}{ Postest } \\
\hline \multicolumn{5}{|c|}{$\begin{array}{l}\text { Ce sont: soit un vide, soit un nuage } \\
\text { ou des "points"(voir figure } 2 \text {.) }\end{array}$} & \multicolumn{5}{|c|}{$\begin{array}{l}\text { Les dessins avec les "particules" } \\
\text { espacées ( représentées par des } \\
\text { points espacés). D'autres types de } \\
\text { dessins (le vide, le nuage) diminuent. }\end{array}$} \\
\hline & points & vide & nuage & autres & & points & vide & nuage & autres \\
\hline $5 \mathrm{E}$ & 1 & 8 & 4 & 2 & $5 \mathrm{E}$ & 2 & 6 & 1 & 6 \\
\hline $5 \mathrm{P}$ & 5 & 5 & 1 & 4 & $5 \mathrm{P}$ & 11 & 1 & 2 & 1 \\
\hline
\end{tabular}

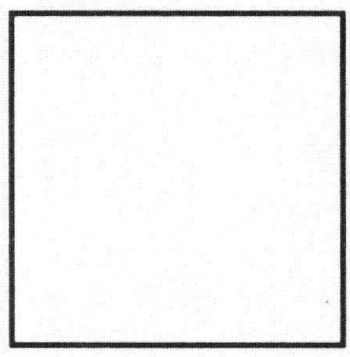

invisible

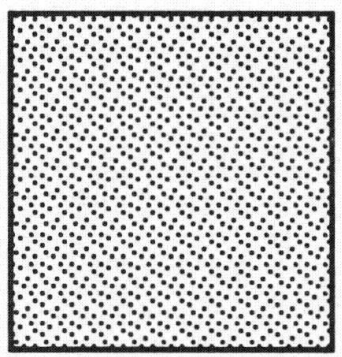

points

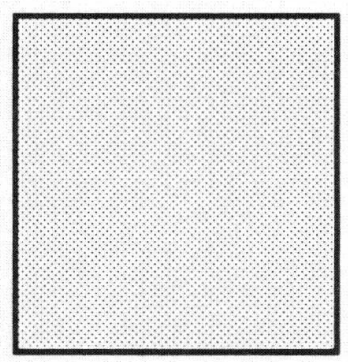

brouillard

Figure 2 : l'air

Tableau VIII. - Résultats comparés du prétest et du postest : définition de la molécule.

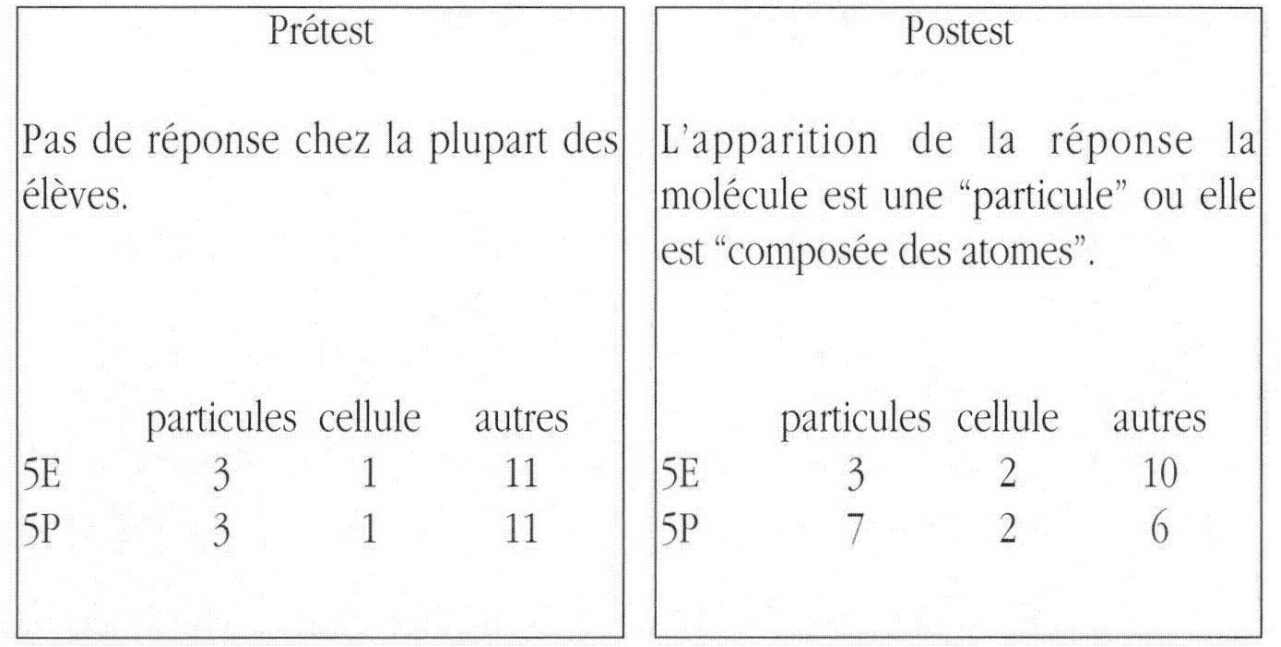

On note que certains élèves assimilent la molécule à la cellule. Ce fait est probablement dû à la contamination de l'enseignement de biologie. Il pourrait expliquer partiellement le phénomène d'animisme que l'on observe dans les représentations. Le phénomène animiste, existant chez la plupart des élèves, est peu modifiable par l'enseignement de chimie; les élèves de 5P notamment ont toujours tendance à croire, après l'enseignement, que la molécule est vivante. Les tableaux suivants montrent qu'il a même tendance à augmenter avec les classes 5P 
Tableau IX - Nombre d'élèves qui pensent que l'atome est vivant dans le prétest et le postest

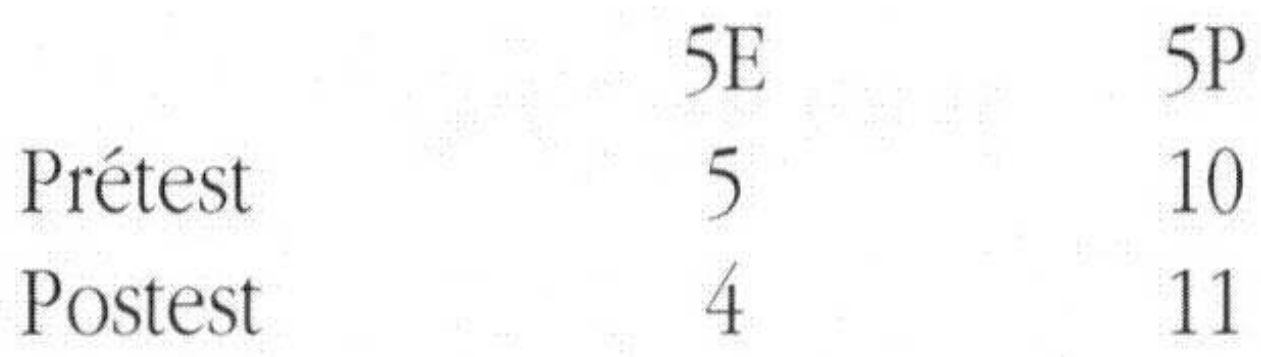

Tableau X.- Nombre d'élèves qui pensent que la molécule est vivante dans le prétest et le postest.

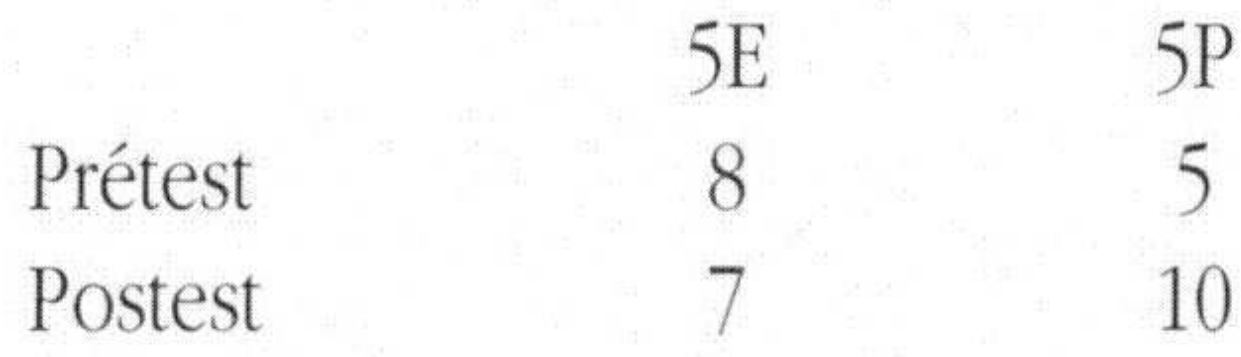

31 Par ailleurs, nous avons voulu savoir si ce phénomène animiste s'atténuait avec l'âge. En classe de 3ème, nous avons obtenus un résultat surprenant : 26 élèves sur 27 interrogés imaginent molécules et atomes vivants.

\section{Conclusion}

On a noté que les performances des élèves sont souvent peu stables. Il arrive qu'ils réussissent à une question donnée dans le prétest mais échouent à la même question dans le postest. Il existe également un décalage du fonctionnement cognitif chez le même élève; c'est-à-dire que les élèves ne généralisent pas ce qu'ils ont apparemment appris pendant le cours (cf. le bilan d'apprentissage), à d'autres phénomènes ( $\mathrm{cf}$. le questionnaire final). Leurs représentations, leurs explications fonctionnent différemment d'un cas à l'autre. Néanmoins, il nous semble que les élèves qui ont subi l'enseignement particulaire (5P) manifesteraient de meilleures acquisitions par rapport à ceux qui ont subi le seul modèle expérimental.

BIBLIOGRAPHIE 


\section{Références}

BRESSON F., La genèse des propriétés des objets, Journal de Psychologie normale et pathologique. 2, p. 143-168,1971.

CAUZINILLE - MARMECHE E. et VEILL BARAIS A., Quelques causes possibles d'échec en mathématiques et en sciences physiques, Psychologie Française, 34-4, pp. 277, 283, 1989.

CHEN L.Y., Approche des processus cognitifs et des représentations mis en œuvre dans l'enseignement de la chimie. Mémoire de maîtrise de psychologie, Université Paul Valéry, Montpellier III.

DOLLE J.M., Ces enfants qui n'apprennent pas, Diagnostics et remédiation, PAIDOS / Centurion, 1989.

LEOMARD F. et BELLANO D., Les conditions d'acquisition d'une nouvelle connaissance, Revue française de pédagogie, 83, pp. 39-46, 1988.

MART1NAND J-L., Connaître et transformer la matière, Peter Lang, Berne, pp.149-174, 1985.

MEHEUT M., Des représentations des élèves au concept de réaction chimique : premières étapes, Bulletin de l'union des physiciens, 716, pp. 997-1011, 1989.

REUCHLIN M., Formalisation et réalisation dans la pensée naturelle : une hypothèse, Journal de psychologie normale et pathologique, 70, pp. 389-408, 1973.

REUCHLIN M., Les différences individuelles à l'école, Presses Universitaires de France, p. 64, 1991. RAULIN P., Construction du concept d'élément chimique : représentation d'élèves de seconde et relation avec quelques obstacles historiques, Mémoire du D.E.A. de Didactique des disciplines scientifiques. Université Claude Bernard-Lyon I. 1991.

\section{RÉSUMÉS}

Nous proposons deux modèles d'enseignement, concernant l'acquisition du concept d'élément chimique en classe de 5ème. Le premier est basé sur une approche expérimentale et empirique, le second introduit les notions de molécules et d'atomes avant la réalisation d'expériences. Les deux groupes ont passé les mêmes questionnaires avant et après application des modèles d'enseignement. Certains résultats semblent inhabituels et nous tentons de les expliquer.

We propose two models of teaching the concept of chemical elements at French level five. The first model is based on an experimental and empirical approach, the second one introduces the notions of molecules and atoms before the experiences. The two groups have been tested by the same questionnaries before and after the application of different teachings. Some results seem unusual and we try, in this paper, to propose an interpretation

\section{INDEX}

Mots-clés : élément chimique, fonctionnement cognitif, modèle expérimental, modèle particulaire 


\section{AUTEURS}

\section{ERICK CAZALET}

Enseignant au collège Antoine Bigot de Nîmes, chargé de cours en psychologie génétique à l' université de Montpellier III

\section{L.Y. CHEN}

Etudiante en maîtrise de psychologie génétique à l'université de Montpellier III 